\title{
ON THE STONE-WEIERSTRASS THEOREM
}

\author{
KUNG-FU NG
}

(Received 2 May 1975)

\begin{abstract}
Let $M$ be a vector subspace of the Banach space $C(\Omega)$ of all real-valued continuous functions on a compact space $\Omega$, and suppose that $M$ contains a subset $L$ and the constant functions. Then in order that $L$ be dense in $M$ it is necessary and sufficient that $L, M$ satisfy a filtering property and that each $m$ in $M$ can be approximated on every two points of $\Omega$ by functions in $L$.
\end{abstract}

In generalizing the classical Weierstrass theorem, one of the usual forms of the so called Stone-Weierstrass theorem reads: Let $L$ be a subset of the Banach space $C(\Omega)$ of real continuous functions on a compact space $\Omega$. Then $L$ is dense in $C(\Omega)$ provided that the following four conditions are satisfied:

(a) $L$ is a subspace of $C(\Omega)$;

(b) $L$ is a sublattice of $C(\Omega)$;

(c) $L$ separates the points of $\Omega$;

(d) $L$ contains the constant functions.

Because of (a) and (d), the condition (c) may be replaced by

(c') Each $f$ in $C(\Omega)$ can be approximated on every (pair of) two points on $\Omega$ by functions in $L$ in the sense that for $\varepsilon>0, w_{1}, w_{2} \in \Omega$, there exists $l \in L$ such that

$$
\left|l\left(w_{i}\right)-f\left(w_{i}\right)\right|<\varepsilon \quad(i=1,2) .
$$

Thus, in the presence of (a), (b) and (d), the two-point approximation property $\left(c^{\prime}\right)$ is sufficient (and necessary) for the uniform approximation (i.e., the density property) of $L$ to hold. On the other hand, neither (a), (b) nor (d) is a necessary condition; for example, the vector subspace of $C[0,1]$ generated by the exponential function and all polynomials of the form $a_{1} x+a_{2} x^{2}+\cdots+a_{n} x^{n}$ is dense in $C[0,1]$ but does not satisfy conditions (b) and (d). Some trivial examples show that (a) is also unnecessary. In this note, we put forward the following result which in particular gives a set of necessary and sufficient conditions for a subset (not necessarily a vector subspace) $L$ to be dense in $C(\Omega)$. 
THEOREM 1. Let $M$ be a vector subspace of $C(\Omega)$, and suppose that $M$ contains a subset $L$ and the constant functions. Then in order that $L$ be dense in $M$, i.e., $M \subseteq \bar{L}$ it is necessary and sufficient that the following two conditions be satisfied:

(a) For each $m \in M$, the family $\{l \in L: l<m\}$ is $\uparrow$ (directed upwards or empty) and $\{l \in L: l>m\}$ is $\downarrow$ for each $m \in M$.

(b) each $m$ in $M$ can be approximated on every two points of $\Omega$ by functions in $L$.

If $L$ is a lattice then (a) certainly holds. Thus the classical Stone-Weierstrass Theorem follows immediately. The theorem is due to Edwards and VincentSmith (1968) in the special case when $L$ is a vector subspace containing the constant functions.

To see the necessity, suppose that $M \subseteq \bar{L}$. Then (b) certainly holds and $1 \in M \subseteq \bar{L}$, so $\exists e \in L$ such that $\|e-1\|<\frac{1}{2}$; then $\frac{1}{2} \leqq e \leqq 3 / 2$ on $\Omega$. To verify (a), let $m \in M$, and

$$
l_{1}, l_{2} \in L_{m}=\{l \in L: l<m\} .
$$

By compactness of $\Omega, \exists \varepsilon>0$ such that $l_{1}, l_{2}<m-2 \varepsilon$. Since $m-\varepsilon \in M$, for this $\varepsilon, \exists l \in L$ such that $\|l-m+\varepsilon\|<\varepsilon$ so $l \in L_{m}$ and $l_{1}, l_{2}<m-2 \varepsilon<l$, as required to prove for the direct property of $L_{m}$. Similarly one can show the other half assertion in (a). The sufficiency of (a) and (b) can be established by modifying arguments of Edwards (1969) together with some standard results in convexity theory. Alternatively, it can be seen immediately from Theorem 2 (below) which is a variant of Edwards theorem and its presentation is more closed to the classical theorem of Stone-Weierstrass.

To facilitate our further discussions, we must recall a few standard concepts in the Choquet boundary theory. We shall identify the Banach space $M(\Omega)$ of real Radon measures on $\Omega$ with the Banach dual of $C(\Omega) . M^{+}(\Omega)$, $P(\Omega)$ respectively denotes the positive Radon and probability measures on $\Omega$. For each $x \in \Omega, \varepsilon_{x} \in P(\Omega)$ is the point-measure concentrated on $x$. Let $W$ be a wedge of upper semi-continuous (u.s.c.) functions on $\Omega$ into [ $-\infty, \infty)$. For $\mu, \nu$ in $P(\Omega)$, we say that $\mu<\nu$ (relative to $W$ ) if $\mu(f) \leqq \nu(f)$ for all $f \in W$. We shall write $\mu \sim \nu$ if $\mu\langle\nu$ and $\mu>\nu$. For each $x$ in $\Omega$, let

$$
[x]_{w} \equiv[x]=\left\{y \in \Omega: \varepsilon_{y} \sim \varepsilon_{x}\right\}=\{y \in \Omega: f(y)=f(x) \forall f \in W\} .
$$

Then, by definition, $x \in \Omega$ is a point in the Choquet boundary $C h_{w}(\Omega)$ if supp $\mu \subseteq[x]$ whenever $\mu>\varepsilon_{x}$. Thus, if $W$ is assumed to separate the points of $\Omega$, then $x \in C h_{w}(\Omega)$ iff $\mu=\varepsilon_{x}$ whenever $\mu \sim \varepsilon_{x}$.

If one takes $W=C(\Omega)$ then clearly $C h_{W}(\Omega)=\Omega$. Another example is to take $\Omega=K$, a compact convex set in a locally convex space and $W=A(K) \subseteq$ $C(K)$ consisting of continuous affine functions, then $C h_{w}(K)$ is precisely the extreme boundary $\partial_{e} K$ of $K$. 
The following theorem is proved by Edwards (1969) in the case when $\Omega=K, M=A(K)$ and $L$ is a vector subspace of $M$.

THeOREM 2. Let $L, M$ be as in Theorem 1, and suppose that condition (a) is satisfied. Let $f \in M$. Then $f \in \bar{L}$ iff it can be approximated on every two points of $C h_{M}(\Omega)$ by functions in $L$, that is, iff,

$\left(^{*}\right) \forall \varepsilon>0, w_{1}, w_{2} \in C h_{M}(\Omega), \exists l \in L$ such that $\left|l\left(w_{i}\right)-f\left(w_{i}\right)\right|<\varepsilon$ for $i=1,2$.

The necessity part is trivial and the idea of proof for the sufficiency part follows essentially Edwards (1969) and is based on a generalized Dini-Cartan filtering argument together with an application of Bauer's [c.f. Alfsen (1971)] maximal principle. For completeness, we give a proof and this proof will be divided into a few steps.

Proposition 1. Let $W$ be as before: a wedge of u.s.c. functions on $\Omega$. Then each $f \in W$ attains its $\Omega$-maximum on the Choquet boundary $C h_{W}(\Omega)$. More generally if $f$ is a pointwise limit of a directed downward subfamily of $W$ then $f$ attains its $\Omega$-maximum on $C h_{w}(\Omega)$.

The first assertion is the usual content of the well-known Bauer's maximal principle. The second assertion can be deduced from the first. In fact, let $W_{-\infty}$ be the set of all pointwise limits of directed downward subfamilies of $W$. Then $W_{-x}$ is also a wedge of u.s.c. functions. By a well-known monotone convergence theorem in integration theory, it is easy to verify that $\mu>\nu$ relative $W$ iff $\mu>\nu$ relative $W_{-\infty}$, thus $C h_{w}(\Omega)=C h_{w_{-\infty}}(\Omega)$, and the result follows.

The following result generalizes the well-known Dini-Cartan theorem:

Proposition 2. Suppose that $W \supseteq \mathscr{G} \downarrow f<-w$ on $C h_{w}(\Omega)$ for some $w \in W$. (More precisely, let $\mathscr{G}$ be a directed downward subfamily of $W$ with pointwise limit $f$ on $\Omega$ such that $f<-w$ on $C h_{w}(\Omega)$.) Then there exists $g_{0} \in \mathscr{G}$ such that $g_{0}<-w$ on $\Omega$.

Proof. By assumption $f+w<0$ on $C h_{w}(\Omega)$ and $f+w \in W_{-\infty}$ (since $W$ is a wedge); it follows from the preceding proposition that $f+w<0$ on $\Omega$. Thus $\mathscr{G} \downarrow f<-w$ on $\Omega$ and it now follows from the classical Dini-Cartan argument that $\exists g_{0} \in \mathscr{G}$ such that $g_{0}<-w$ on $\Omega$.

Proposition 3. Let $M$ be a vector subspace of $C(\Omega)$ containing the constant functions. For each $h \in C(\Omega)$, define the upper envelope function $\hat{h}$ by

$$
\hat{h}(x)=\inf \{m(x): m \in M, h<m \quad \text { on } \Omega\} \quad(x \in \Omega) .
$$

Then, for each $x \in \Omega$, one has that

$$
\hat{h}(x)=\max \left\{\mu(h): \mu \in P(\Omega), \mu \sim \varepsilon_{x} \text { relative to } M\right\} .
$$

In particular, if $h$ is constant on $[x]_{M}$ and $x \in C h_{M}(\Omega)$ then $\hat{h}(x)=h(x)$. 
Proof. For any fixed $x \in \Omega$, the map $h \leadsto \hat{h}(x)$ is a welldefined sublinear functional on $C(\Omega)$ such that $\hat{h}=h$ if $h \in M$ and $\hat{h} \leqq 0$ if $h \leqq 0$. For a fixed $h \in C(\Omega)$, by the Hahn-Banach theorem there exists a linear functional $\phi$ on $C(\Omega)$ such that $\phi(h)=\hat{h}(x)$ and $\phi(f) \leqq \hat{f}(x)$ for each $f$ in $C(\Omega)$. Then $\phi$ is a positive normalized linear functional on $C(\Omega)$ (so $\phi \in P(\Omega)$ ) and $\phi \sim \varepsilon_{x}$ relative to $M$. Hence

$$
\hat{h}(x)=\phi(h) \leqq \max \left\{\mu(h): \mu \in P(\Omega), \mu \sim \varepsilon_{x} \text { relative to } M\right\},
$$

and the result follows since the opposite inequality is easy to check.

We are now ready to prove the sufficiency part of Theorem 2. Suppose then $L, M$ and $f$ satisfy the condition (a) and the two-point approximation property $\left(^{*}\right)$. We wish to show that $f \in \bar{L}$. Let $\varepsilon>0$ be given. For each $x \in C h_{M}(\Omega)$, it follows from (*) that the family

$$
L_{x}=\{l \in L: l(x)<f(x)+\varepsilon\}
$$

is non-empty and has the pointwise supremum, say $f_{x}$, such that $f-\varepsilon<f_{x} \leqq$ $+\infty$ on $C h_{M}(\Omega)$. If we can show that $L_{x}$ is directed upward then proposition 2 would enable us to choose $l_{x} \in L_{x}$ such that $f-\varepsilon<l_{x}$ on $\Omega$ and consequently the family

$$
\mathscr{G}=\{l \in L: f-\varepsilon<l \text { on } \Omega\}
$$

is non-empty and has the pointwise infimum $<f+\varepsilon$ on $C h_{M}(\Omega)$. By condition (a) it follows from Proposition 2 that there exists $g \in \mathscr{G}$ such that $g<f+\varepsilon$ on $\Omega$. Then $g \in L$ and $\|g-f\|<\varepsilon$. Thus, to complete the proof one need only show that $L_{x}$ is directed upward for all $x \in C h_{M}(\Omega)$. Accordingly, let $l_{1}, l_{2} \in L_{x}$, $h=l_{1} \vee l_{2}$ and $\alpha=f(x)+\varepsilon$. Then, in virtue of Proposition 3, $\hat{h}(x)=h(x)<\alpha$. It follows from the definition of $\hat{h}$ that there exists $m \in M$ such that $h<m$ and $m(x)<\alpha$. Now, $l_{1}, l_{2}$ are elements of the family $\{l \in L: l<m\}$ which is directed upward by condition (a); thus there is $l_{3} \in L$ 'such that $l_{1}, l_{2} \leqq l_{3}<m$ (so that $l_{3}(x)<m(x)<\alpha$ ). This implies that $l_{3} \in L_{x}$ and $l_{3} \geqq l_{1}, l_{2}$, as required to prove.

\section{References}

E. M. Alfsen (1971), 'Compact convex sets and boundary integrals', Springer-Verlag.

E. Bishop and K. de Leeuw (1959), 'The presentation of linear functionals by measures on sets of extreme points', Ann. Inst. Fourier (Grenoble) 9, 305-331.

D. A. Edwards and G. Vincent-Smith (1968), 'A Weierstrass-Stone theorem for Choquet simplexes', Ann. Inst. Fourier (Grenoble) 18, 261-282.

D. A. Edwards (1969), 'On uniform approximations of affine functions on compact convex sets', Oxford Quarterly J. 20, 139-142.

Department of Mathematics

United College

The Chinese University of Hong Kong. 